
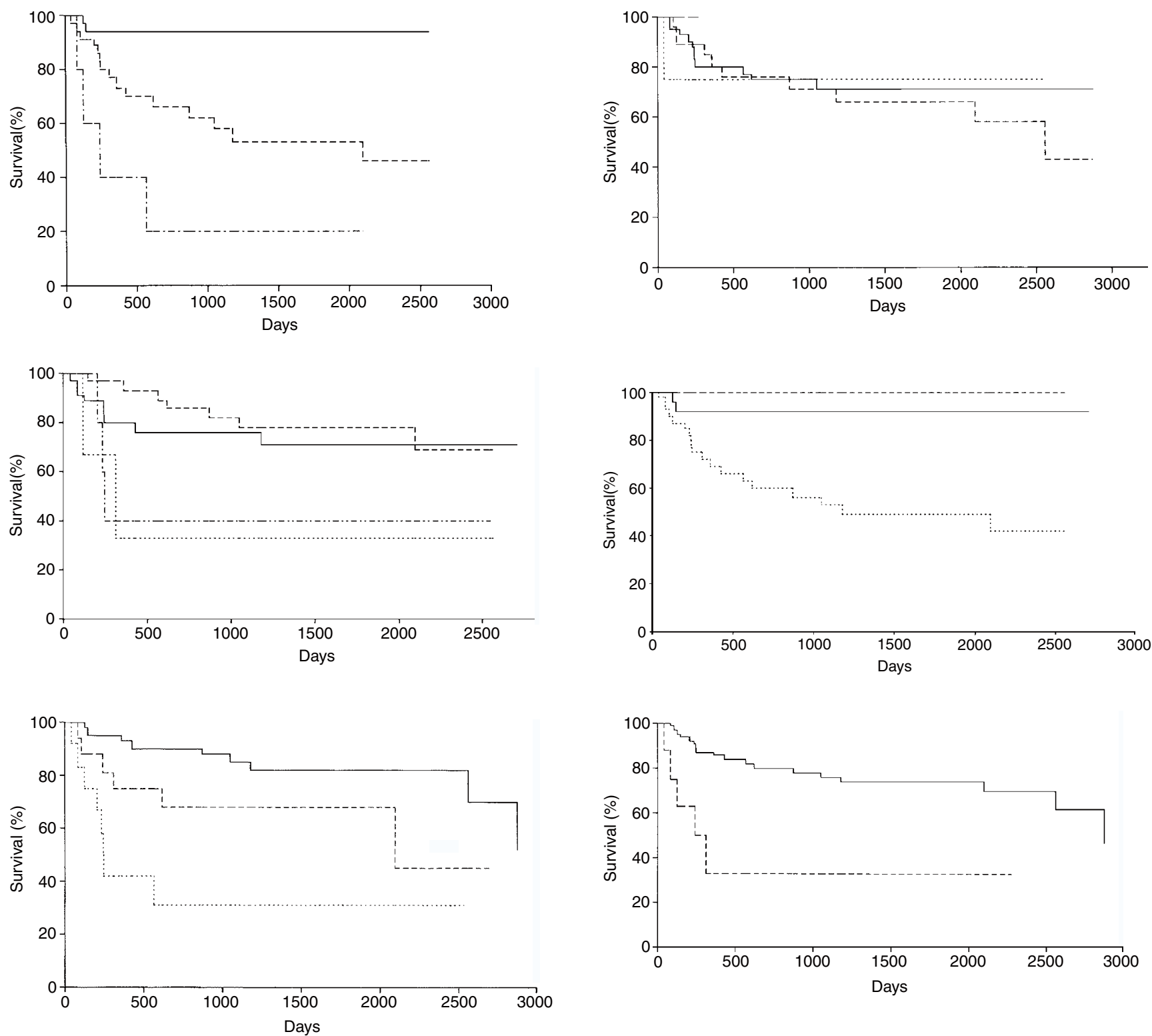

Figure 2 (A) The survival for 81 patients with metastatic TGCT according to the initial postsurgical S-LD-1 $(-=39$ patients with S-LD-1 $<112$ U L-1, $--=37$ with S-LD-1 $112-1120 \mathrm{U}^{-1}$, and.$--=$ five with S-LD-1 $\left.>1120 \mathrm{U} \mathrm{I}^{-1}\right)$. (B) The survival according to S-hCG $\left(\frac{1}{-1}=39\right.$ patients with S-hCG $<30$ IU $\mathrm{I}^{-1}$ $--=33$ with S-hCG 30-4999 IU $\mathrm{I}^{-1}, \ldots=$ four with S-hCG 5000-50 $000 \mathrm{IU} \mathrm{I}^{-1}, \ldots=$ five with S-hCG $>50000 \mathrm{IU} \mathrm{I}^{-1}$ ). (C) The survival according to the risk groups of the IGCCCG classification ( $-=49$ patients with a good prognosis, $-=16$ with an intermediate prognosis, and $---=16$ with a poor prognosis).

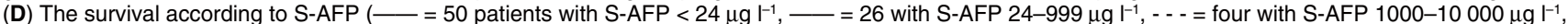
$--=$ one with S-AFP $>10000 \mu \mathrm{g} \mathrm{l^{-1 }}$. (E) The survival according to the concomitantly normal and raised levels of S-LD-1 and S-LD $(-=31$ patients with normal values of S-LD-1 and S-LD, $--=$ eight with a normal value of S-LD-1 and a raised S-LD, and - - - = 42 with a raised S-LD-1 (with or without a raised S-LD)). (F) The survival according to the S-LD-1/S-LD ratio $(-=73$ patients with an S-LD-1 ratio $<0.375$, and $--=$ eight with a ratio $>0.375)$

doi: 10.1054/ bjoc.2000.1450, available online at http://www.idealibrary.com on IDE

\title{
Serum lactate dehydrogenase isoenzyme 1 as a prognostic predictor for metastatic testicular germ cell tumours - reply
}

\section{Sir}

von Eyben et al describe their experience of splitting total LDH into its various isoenzymes and have identified LDH isoenzyme 1 (S-LD-1) as having prognostic significance in untreated patients with metastatic germ cell tumours. The evidence they present is clearly impressive and S-LD-1 appears to be able to identify patients with a poor outcome as effectively as the IGCCCG specification or serum HCG alone. 
We agree that validation of their findings with a larger set of patients would be useful and also feel that the prognostic role of S-LD-1 in patients who relapse should also be assessed to see if its predictive role in this setting is maintained.
$J$ Shamash

Department of Medical Oncology, St. Bartholomew's Hospital, Ist Floor King George V Building, West Smithfield, London ECIA 7BE, UK 